\title{
CPHA and Global Health Governance
}

CPHA had the opportunity, through the auspices of the World Federation of Public Health Associations (WFPHA), to participate at this year's $63^{\text {rd }}$ World Health Assembly. This event brought together delegations from over 190 World Health Organization (WHO) member states, as well as representatives from over 100 NGOs, foundations, corporations and other interested parties. This annual event represents one of the most important global health governance events, as it is the one time each year when WHO Member States take decisions that define internationally accepted norms, standards and regulations designed to improve and protect the public's health around the world.

As noted by Dr. Margaret Chan, WHO's current Director General, consensus and agreement was reached on several critical global public health issues, including a code of practice on the international recruitment of health personnel. The WHA, through intense negotiation sessions, some of which lasted all night, also discussed and tackled other important global issues, including food safety, counterfeit medical products, the monitoring of the health-related Millennium Development Goals, strategies to reduce the harmful effects of alcohol, the implementation of the International Health Regulations, and issues related to the rise of chronic non-communicable diseases, which included the issue of marketing of non-alcoholic beverages and foods to children.

CPHA participates in global health governance as well through its role within the WFPHA. Founded in 1967, the WFPHA is the global civil society for public health, bringing together national and regional public health associations from over 60 countries. CPHA became a member in 1973, and has played an important role over the subsequent 37 years. CPHA has served on the WFPHA Executive Board on several occasions, and both Gerry Dafoe and Margaret Hilson, as CPHA representatives to the WFPHA, have served as Federation President.

The Association continues to play an important role within the WFPHA. In April 2009, CPHA attended and supported the participation of representatives from over 13 national public health associations at the $12^{\text {th }}$ WFPHA World Public Health Conference, held in Istanbul (Turkey). Over the past year, CPHA took responsibility to establish and chair the WFPHA Finance Committee and also contributed to the revitalization of the Federation's Tobacco Control Working Group.

CPHA will continue to contribute to the Federation's development and expansion. At its $44^{\text {th }}$ Annual General Meeting on May $16^{\text {th }}$, WFPHA members elected CPHA, represented by Jim Chauvin (Director of Global Health Programs and Policy), as the Federation's Vice-President and President-Elect. CPHA will hold this position for 2 years and will assume the Presidency of the WFPHA in 2012 for a 2-year mandate. CPHA intends to help build and nurture a strong global civil society voice through the Federation's member public health associations in support of priority public health issues, including effective global health governance.

\section{Canadian Public Health Association Board of Directors • Conseil}

Chair/Président

Chair-Elect/Présidente désignée

Chief Executive Officer/

Chef de la direction (ex officio)
Cordell Neudorf, BSC, MD, MHSC, FRCPC Erica di Ruggiero, BSC, MHSC, RD

Debra Lynkowski, LIB

\section{L'ACSP et la gouvernance mondiale de la santé}

Cette année, sous les auspices de la Fédération mondiale des associations de la santé publique, l'ACSP a eu l'occasion de participer à la 63e Assemblée mondiale de la Santé. L'événement a réuni les délégations des plus de 190 États membres de l'Organisation mondiale de la santé (OMS) et des représentants d'une centaine d'ONG, de fondations, de sociétés et autres intéressés. Cette assemblée annuelle est l'un des plus grands rendezvous de la gouvernance mondiale de la santé; c'est en effet le seul moment de l'année où les États membres de l'OMS prennent des décisions qui définissent les normes et les règlements internationaux qui visent à améliorer et à protéger la santé du public autour du monde.

Comme l'a indiqué $\mathrm{D}^{\mathrm{re}}$ Margaret Chan, directrice générale de l'OMS, plusieurs dossiers névralgiques en santé publique mondiale ont fait l'objet d'accords et de consensus, y compris un code de pratique mondial pour le recrutement international des personnels de santé. Au fil de séances de négociation intensives dont certaines ont duré toute la nuit, l'Assemblée mondiale de la Santé a aussi débattu et abordé d'autres grands dossiers internationaux. Il a été question de la salubrité des aliments, des produits médicaux de contrefaçon, de la surveillance des objectifs du Millénaire pour le développement liés à la santé, des stratégies de réduction des effets néfastes de l'alcool et de la mise en œuvre des Règlements sanitaires internationaux. On a aussi abordé certains enjeux liés à la hausse des maladies chroniques non transmissibles, dont le marketing des aliments et des boissons non alcoolisées auprès des enfants.

L'ACSP participe aussi à la gouvernance mondiale de la santé en tant que membre de la Fédération mondiale des associations de la santé publique. Fondée en 1967, la Fédération est la société civile mondiale de la santé publique; elle rassemble les associations pour la santé publique nationales et régionales de plus de 60 pays. L'ACSP en est devenue membre en 1973 et y joue un grand rôle depuis 37 ans. Elle a siégé au bureau de la Fédération à plusieurs reprises, et ses deux représentants, Gerry Dafoe et Margaret Hilson, en ont assuré la présidence.

L'Association continue de jouer un grand rôle au sein de la Fédération. En avril 2009, l'ACSP a assisté au $12^{\text {e }}$ Congrès mondial de santé publique à Istanbul (Turquie) et a soutenu la participation de représentants de plus de 13 associations pour la santé publique nationales. L'an dernier, l'ACSP a eu la responsabilité d'établir et de présider le Comité des finances de la Fédération, et elle a contribué à revitaliser son groupe de travail antitabac.

L'ACSP continuera à œuvrer pour le développement et l'expansion de la Fédération. Lors de leur 44 e Assemblée générale annuelle le 16 mai, les membres de la Fédération ont élu l'ACSP, représentée par Jim Chauvin (directeur du Service des politiques et des Programmes de santé mondiale), au siège de vice-président et de président désigné. L'ACSP occupera ce siège pendant deux ans et assurera la présidence de la Fédération en 2012 pour un mandat de deux ans. Elle entend contribuer à faire retentir haut et fort la voix de la société civile, par le biais des associations pour la santé publique membres de la Fédération, pour faire avancer les priorités en santé publique. Une bonne gouvernance mondiale de la santé est l'une de ces priorités.
Directors/Directeurs

Hope Beanlands, PhD, MPA, MN, RN Brian Bell, MSW

Joel Finlay, MPH

Alycia Fridkin, MHSc
John M. Maxted, MD, MBA, CCFP, FCFP Lynn Mclntyre, MD, MHSc, FRCPC Shannon Turner, BA, BSc, MSc 